\title{
Online Learning System to Help People with Developmental Disabilities Reinforce Basic Skills
}

\author{
Lourdes M. Morales-Villaverde ${ }^{1}$, Karina Caro ${ }^{2}$, Taylor Gotfrid ${ }^{1}$, and Sri Kurniawan ${ }^{1}$ \\ ${ }^{1}$ Baskin School of Engineering \\ University of California, Santa Cruz \\ 1156 High Street \\ Santa Cruz, CA 95064 \\ \{lommoral, tgotfrid, skurnia\}@ucsc.edu \\ ${ }^{2}$ Department of Computer Science \\ CICESE \\ Carretera Ensenada-Tijuana 3918 \\ Ensenada, B.C. 22860, Mexico \\ karicaro@cicese.edu.mx
}

\begin{abstract}
We present the development and evaluation of an online learning system for people with developmental disabilities (DD) of all ages in collaboration with Imagine! and Hope Services, two not-forprofit organizations that provide care services to people with DD. The system was implemented as an HTML5-based web application for iPad. It includes activities that aim to support and improve the process through which people with DD of all ages reinforce basic skills such as recognizing numbers, letters, money, shapes, and colors. User evaluations suggest that a system such as ours will be: 1) helpful in supporting people with DD reinforce basic skills, and 2) well-received by users.
\end{abstract}

\section{CCS Concepts}

- Human-centered computing $\rightarrow$ Accessibility $\rightarrow$ Accessibility design and evaluation methods.

\section{Keywords}

Developmental disability; basic skills; online learning; web application; iPad.

\section{INTRODUCTION}

People often take for granted the fact that they possess basic skills (e.g., recognizing numbers, colors, money) that they learned at a young age and now help them perform daily-living activities. For people with developmental disabilities (DD), performing dailyliving activities can be a struggle, especially if they have not mastered the aforementioned basic skills. Consequently, people with DD often rely on other people, such as those in charge of their care, to carry out those activities. This limits these individuals' participation in valued and meaningful societal roles (e.g., adult, citizen, employee) and reduces their overall life satisfaction $[2,4,9]$. Also, this dependency on others increases their caretakers' workload.

Permission to make digital or hard copies of all or part of this work for personal or classroom use is granted without fee provided that copies are not made or distributed for profit or commercial advantage and that copies bear this notice and the full citation on the first page. Copyrights for components of this work owned by others than ACM must be honored. Abstracting with credit is permitted. To copy otherwise, or republish, to post on servers or to redistribute to lists, requires prior specific permission and/or a fee. Request permissions from Permissions@acm.org.

ASSETS '16, October 23-26, 2016, Reno, NV, USA.

(C) 2016 ACM. ISBN 978-1-4503-4124-0/16/10 . \$15.00.

DOI: http://dx.doi.org/10.1145/2982142.2982174
Technology designed to support people with DD can provide them with new opportunities in terms of meaningful societal roles and positive outcomes (monetary and self-confidence) where they succeed in assuming one or more of those roles $[1,2,3,4,6,10]$.

Before the advent of touchscreen devices, such technologies included "picture prompting" [1, 3, 6], which involved giving individuals with DD pictures that depict how to complete each step required to complete a task, and video-based technologies that involved showing people with DD a video depicting how to complete a task in general (i.e., "video modeling") or step-by-step (i.e., "video prompting") $[1,2,4,8,9]$. The study by Lancioni et al. in [6] discussed two types of picture prompting systems designed to help people with DD perform "vocationally relevant" tasks: first, a physical card-based system with cards containing pictorial descriptions of instructions, and second, a computerbased system where pictorial instructions were shown on a portable device (i.e., a computerized card-based system). Their results suggest that most users had a higher percentage of correct steps with the computer-based system and that they preferred that system to the physical card-based one [6]. Similarly, Furniss et al. discussed their work on a computer-based picture prompting system, VICAID, designed to do two main things: first, enable individuals with DD to complete tasks (related to real work settings) by providing them access to pictorial instructions on how to complete the tasks; and second, alert and engage the job coach or supervisor of the individual with DD when his/her interaction with the system suggests that he/she is having difficulties [3]. Their results, like those of the work discussed in [6], suggest that computer-based systems were more effective than card-based systems in helping users with DD maintain accurate taskperformance (in real-world work settings) and that most users preferred the computer-based systems [3].

Alberto et al. discussed the use of picture prompting and video modeling for teaching individuals with DD how to accomplish daily-living activities such as drawing money from an ATM and purchasing items using a debit card [1]. Ultimately, they found that both technologies were effective and efficient at teaching those skills. Similarly, in [2] Canella-Malone et al. researched the use of video modeling and video prompting technologies when teaching adults with DD daily-living skills such as setting up a table and putting away groceries. Their findings, unlike those of [1], suggest that video prompting was more effective than video modeling, and that certain video attributes (e.g. duration, number of clips, and perspective from which it is filmed) may influence the effectiveness of these technologies as teaching tools. Still, in [4] Goodson et al. found that not all individuals with DD learn from video prompting alone and that the addition of a video-based 
error correction procedure that plays the same clips as the video prompting when the user makes a mistake may help.

Although there are many works on the design of technology to support people with DD perform tasks, especially in employment and independent-living settings (e.g. setting up a table), there is not much work on developing technology that helps people with DD reinforce the basic skills they need to complete those tasks. For example, if an individual is given the instruction: "Place the number two screw on panel A." Then he/she must be able to recognize both numbers and letters to complete it.

The advent of touchscreen devices has spurred development of applications (apps) designed to teach and train users and to supplement rehabilitation therapy of users with all kinds of disabilities. Kagohara et al. conducted a review of works that involved the use of an iPad or iPod Touch as a learning tool for individuals with DD, which suggests that these devices are viable aids for people with DD [5].

The main problem is most of the apps that provide lessons on the basic skills are normally geared towards children. For example, the LetterSchool ${ }^{1}$ iPad app (Figure 1, left) is an app that aims to teach letters to children aged 2-5. As Figure 1 shows, the app has very busy screens with lots of bright colors, images, and objects that move, which in addition to being childish, can be highly distracting to users with DD. Another example is the Learning Gems - Colors N Shapes ${ }^{2}$ iPad app, which is designed to teach shapes and colors to children aged 2-6 (Figure 1, right). This app is more simplistic than the LetterSchool app, but it is also vibrantly colored and has a relatively busy design that may be inappropriate for adults with DD. Previous work also suggests that technology designed for children is generally not well-received by adults who acquired a cognitive disability (CD) (e.g., as a result of stroke) since they feel it is not appropriate for them as adults [7].

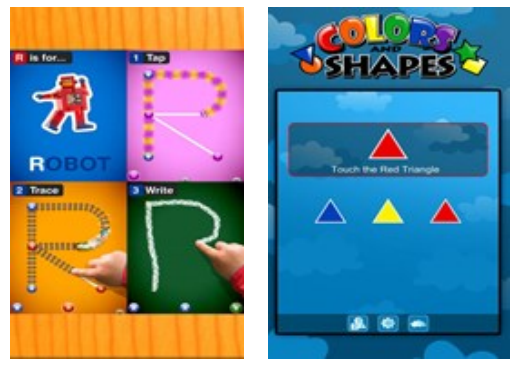

Figure 1. LetterSchool app (left) and Learning Gems - Colors N Shapes app (right).

The purpose of the work reported in this paper is to develop and evaluate an online learning system that provides individuals with DD of all ages activities that can reinforce basic skills. Working in collaboration with Imagine! and Hope Services, two not-for-profit organizations that provide support services to people with DD, we iteratively created and evaluated personas, storyboards, wireframes, and prototypes. The system includes seven activities that help users review numbers, colors, shapes, lowercase and uppercase letters, and U.S. currency; and practice gathering a set amount of money. The activities were designed to be simple, clear, rewarding, and appropriate for users with DD of all ages.

This paper is structured as follows: First, we give an overview of our methods for gathering system requirements and present a

\footnotetext{
${ }^{1}$ LetterSchool

${ }^{2}$ Learning Gems - Colors N Shapes
}

subset of the requirements gathered, specifically those related to the features evaluated with primary users (individuals with DD). Secondly, we present the general design characteristics of the high-fidelity prototype of the system. Next, we give an overview of how we ran preliminary evaluations of our system and the main changes that resulted from those evaluations. Then, we discuss how we evaluated a high-fidelity prototype of the system through a comprehensive user study along with the results from that study. Finally, we present our conclusions along with future work.

\section{METHODS}

\subsection{Gathering System Requirements}

Working in collaboration with Imagine! employees and caregivers through focus group sessions, we gathered the system requirements (functional and non-functional) for the proposed system by iterating over the creation and evaluation of personas, usage scenarios, storyboards, wireframes, and prototypes.

Through the initial focus group sessions, we gathered information about our target users' desires in terms of what they would want out of the proposed system (e.g. the types of skills they want to work on), motivations for improving their skills (e.g. to be more independent), and activities of interest (e.g. activities where users interact with iPads or work on learning or improving basic skills that our proposed system is intended to help with), along with their surroundings during those activates (e.g. the people who are around them while they do those activities).

During this time, we noted that many of Imagine!'s clients had iPads (they were covered by their health insurance under the notion that they are needed for rehabilitation therapy). We also found previous work that supports that iPads are viable technological devices for teaching individuals with DD skills such as cooking and cleaning [5]. Hence, we decided to develop our proposed system as an iPad app and, as a first step, conducted a competitive analysis of existing iPad apps that could be used to help people with DD learn or improve the skills that our proposed system is intended to help with. We found that most of the apps that could help, like the LetterSchool and Learning Gems - Colors $\mathrm{N}$ Shapes apps, were geared towards children. As we explained before, using apps geared towards children to teach basic skills to individuals with DD can be problematic because: 1) their design normally includes elements that may be too distracting or overwhelming for adults with DD (e.g. too many bright colors, loud noises, animations), and 2) their strategy for motivating users to complete tasks may be appropriate for children but not necessarily appropriate for older individuals with DD (i.e., it may not be as motivating or as engaging for them).

Once we had established a preliminary set of system requirements for our proposed system, we created personas, usage scenarios, storyboards, wireframes, and low-fidelity prototypes; which guided the next round of focus group sessions with our collaborators. Those focus group sessions were centered on what the system should do, look and act like (i.e., its functionalities, interface, and complexity) in order for it to meet users' abilities and needs. Based on the feedback from the focus group sessions, we iteratively created low-fidelity prototypes (Figure 2, next page), which ranged from drawings on a white-board to slideshow presentations, and improved the system requirements until they were both accepted as a basis for a final prototype. As Figure 2 shows, the design evolved in many aspects, like the color choice for the background and highlight, and the size of the numbers. 


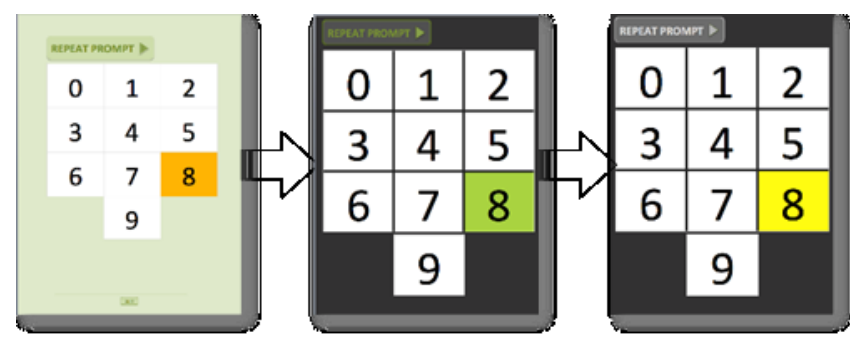

Figure 2. Iterations of activity on recognizing numbers.

\subsection{System Requirements}

Before presenting the system requirements, it is important to note:

1. Our primary users (users) are individuals with DD and their caregivers are the secondary users (administrators).

2. Typical usage scenarios depend on users' cognitive abilities:

a. The two typical usage scenarios for users with limited cognitive abilities are: a) An administrator selects the activity that the user will attempt and helps the user attempt the activity, and b) An administrator checks the user's progress in previously attempted activities in order to assess the user's needs and decide which activity the user should attempt next.

b. Otherwise, the typical usage scenario is: a user selects and attempts an activity independently.

The results of the above exercises are a list of functional and nonfunctional requirements, and their priorities and reasons as given by our stakeholders.

\subsubsection{Functional Requirements}

\subsubsection{High Priority}

1. Include activities that help people with DD review basic skills such as recognizing letters, money, colors, shapes, and numbers. Reason: Many daily-living activities require such basic skills.

2. Give users positive reinforcers ${ }^{3}$ when they follow prompts (e.g., "touch number $\mathrm{x}$ ") correctly during the activities. Reason: Positive reinforcers must serve as positive feedback and as an incentive to continue doing the activities.

3. When users do not follow a prompt correctly during an activity, give them various attempts (up to three) and hints on how to follow prompts correctly along with learning reinforcers ${ }^{4}$ which also help users learn the correct response. On the last attempt, give users no other choice but to follow the prompt correctly and learn the correct response. Reason: In general, the system should not give users negative feedback. Also, the system should help users learn from their mistakes and not punish them.

4. Give users 90 seconds to respond to a prompt before issuing a timeout and proceeding as if an incorrect response was made or, if on the last attempt, ending the activity. Reason: The system should ensure that if users are distracted by other activities, they are informed that the system is still waiting for their interaction before terminating the session.

\subsubsection{Low Priority}

5. Display progress information to users during activities. Reasons: Users should know which round they are on out of the total number of rounds in an activity.

${ }^{3}$ Positive reinforcer: An image, video, or audio clip that users may enjoy seeing or hearing.

${ }^{4}$ Learning reinforcer: A visual cue (e.g. highlight) or audio clip that aims to help users learn.

\subsubsection{Non-functional Requirements}

\subsubsection{High Priority}

1. System should be computer-based and compatible with the ever-changing versions of iPad and iOS. Reasons: As mentioned before, previous work suggests that people with DD tend to prefer computer-based systems to paper-based ones $[3,6]$ and that iPads are aids for individuals with DD basic skills [5]. Plus, many of Imagine!'s clients had iPads and thus, some knowledge of their operation.

2. The system (specifically the activities) should: a) avoid distracting users (e.g., avoid using unnecessary sounds or buttons); b) be age-appropriate (i.e., not too childish for adults and not too complex for children to use); and c) use high-contrast and color-blind friendly colors, large fonts and buttons, and ample input space. Reason: The system (specifically the activities) should be usable, accessible and age-appropriate for people with DD of all ages who may have other disabilities (i.e., users may have limited cognitive, motor, and/or visual skills).

\subsubsection{Medium Priority}

3. System shall be easily maintainable and have an expected usage life of 2-3 years. Reason: The system should not increase caregivers' workload by requiring maintenance.

\section{High-fidelity Prototype}

Based on the system requirements that we gathered, we developed a high-fidelity prototype. Following the same process as with our low-fidelity prototype, we iterated over the prototype's design based on the feedback we got from focus group discussions with our collaborators. The main design characteristics of the final high-fidelity prototype of the proposed system are as follows:

- It was implemented as an HTML5 web-app for iPad so that it has a longer lifespan and is easier to maintain than a native iOS/iPad app. It also works on most desktop web browsers.

- It offers seven activities: Money Addition (MA), Money, Numbers, Lowercase Letters (LL), Uppercase Letters (UL), Shapes, and Colors. These consist of a set of prompts that aim to help users reinforce a basic skill while assessing their knowledge on that skill. In general, they help users practice gathering money (Figure 3, left) or recognize the following by name: U.S. currency (bills and coins most commonly used), numbers, letters in uppercase and lowercase, common shapes (Figure 3, right), and primary colors. The activities were designed to mimic how users normally perform such activities (i.e., activities where they reinforce the corresponding skills) at Imagine!.

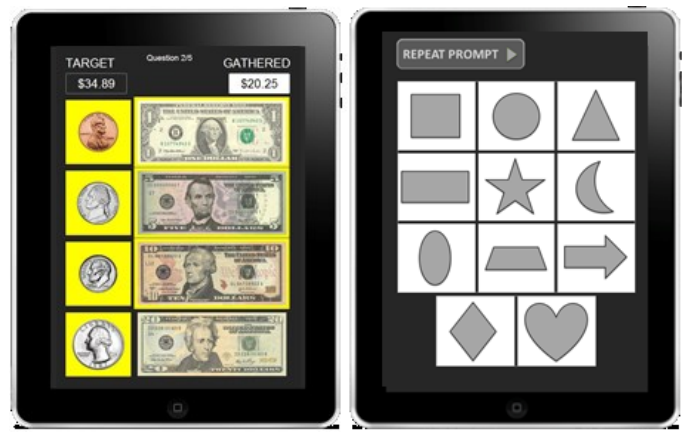

Figure 3. Money Addition (left) and Shapes (right) activities.

- Users respond to prompts by touching one of the options shown. For example, in the Numbers activity a grid is shown 
with all the numbers from 0 to 9 and users are prompted to touch a (randomly selected) number. The Money, Shapes, Colors, UL, and LL activities behave similarly.

- In the MA activity (Figure 3, left) a grid with the main bills and coins that make up the U.S. currency is shown (as in the Money activity) and users are asked to add those up (by touching them) to reach a target total (between $\$ 0.01$ and $\$ 100)$ that is randomly generated in each of the five rounds that make up the MA activity. Note that, the currency that can be used to add up to the target total is highlighted in yellow. For example, if the user needs to reach $\$ 34.89$ and has already gathered $\$ 20.25$, then the twenty-dollar bill is not highlighted while the rest of the currency is (Figure 3, left). Although the goal of this activity is for users to reach the target total with the minimum amount of bills and coins (i.e., the minimum number of presses), reaching the target total will be noted as a success despite the total number of presses. That way the activity fulfills its purpose without giving users negative feedback. Likewise, when a user presses currency that is not highlighted, it is not marked as an error and the gathered amount is not changed.

- The Money and MA activities display images of real U.S. currency (Figure 3, left) instead of drawings to help users with recognition in real-life.

- The numbers and letters in the Numbers, UL, and LL activities are displayed in a random order.

- When users make a selection (right or wrong) after a prompt, they are given a learning reinforcer: an audio clip saying the name of the selection and, if the selection has another name, the other name too (e.g., "A penny, one cent.").

- When users do not follow a prompt correctly, all the activities (except the MA activity) give users three more attempts and hints on how to follow the prompt correctly (e.g., highlighting the correct choice and/or removing the 'incorrect' options). Hints get more direct with each attempt. Note that, while these techniques of prompting along with error-correction have proven to be effective at helping people with DD perform tasks $[1,2,3,4,6]$, the main goal of the activities is to teach users the correct choice while helping them follow prompts correctly. That is why, besides the hints, the activity gives users the learning reinforcers. In general, the way the activities behave in this regard is:

- When the user does not follow a prompt correctly on the first screen (i.e. on the first attempt), the second screen is shown with the correct choice highlighted in yellow (Figure 4(b)) or, as in the Colors activity, with black/white dashes. Then, the prompt is repeated.

- When the user does not follow a prompt correctly on the second screen, the third screen is shown with the correct choice highlighted and the incorrect options are hidden (Figure 4(c)). Then, the prompt is repeated.

- When the user does not follow a prompt correctly on the third screen, the last screen is shown with the correct choice (still highlighted) taking up most of the screen (Figure 4(d)) and the prompt is repeated.

- When users follow prompts correctly, they are given two positive reinforcers: an audio clip saying "Good Job!" or "That's right!" and a picture of something they might like. Since we were evaluating the prototype primarily with Imagine!'s clients, the pictures were of their favorite football team (the Denver Broncos). We used the same images when evaluating an improved prototype with Hope Services' clients, and found that pictures of players from a popular sport work as positive reinforcers in general.

- Activities were designed to be simplistic, clear, and accessible by: 1) avoiding unnecessary buttons, sounds, and distracting objects; 2) making the activity the focus and the prompts and interface straightforward; 3) highlighting with colorblind-friendly colors (yellow), and using a high-contrast color-scheme (white on black), practical fonts (e.g., easily readable), and good quality images to make them accessible to users with visual impairments; 4) making choices (i.e., the numbers, shapes, colors, and images of objects) sufficiently large to make them accessible to users with visual and motor impairments; and 5) allowing users to provide input by just tapping the screen (no dragging or swiping) to make them accessible to users with motor disabilities.
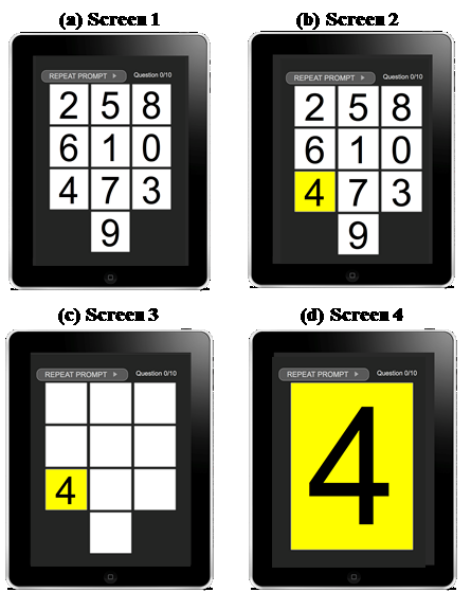

Figure 4. Screens for the Numbers activity.

\section{Evaluations and Results}

In this paper, we focused primarily on evaluating the activities for primary users (individuals with DD) even though we have implemented other features for administrators (e.g., data collection of users' progress). We started by conducting heuristic evaluations of the activities at the beginning stages of development to test their usability and UI. Based on those evaluations, we improved the prototype and the list of requirements based on those evaluations. After getting IRB approval, we conducted preliminary user evaluations of the prototype, specifically the activities, in collaboration with Imagine!, with some of their clients with DD at their facilities. Based on the preliminary user evaluations, we further improved the prototype and the study. Then, in collaboration with Hope Services, we ran a more comprehensive user study with ten of their clients with DD at one of their facilitates.

\subsection{Preliminary Evaluations and Results}

We first ran preliminary evaluations of the prototype, specifically the activities, through heuristic evaluations. We chose to test the compliance of the system's UI design with Nielsen's 10 Usability Heuristics 5 since they are widely accepted and they cover all the usability properties that we want our UI design to comply with. Based on Nielsen's 10 Usability Heuristics and the usage scenarios we constructed when gathering system requirements, we developed a set of tasks for evaluators to perform in order for them to examine the UI design and usability of the system.

${ }^{5}$ http://www.nngroup.com/articles/ten-usability-heuristics/ 
Fifteen evaluators (not the authors) were asked to perform those tasks (with every activity) and judge the UI design's compliance with the chosen usability principles. Evaluators were between the ages of 21 to $28($ mean $=23)$ and they all had experience with iOS devices (e.g., iPad, iPhone), UI and heuristics evaluation. Based on these evaluations, we made the following improvements:

- Fixed timing for the transition between screens and the audio-based positive reinforcers so audio would not overlap.

- Progress information was added while the activity loads (i.e., the message "Loading" is shown while the activity loads the necessary graphics and audio) and while it is in progress (e.g., it specifies which round the user is on out of the total number of rounds that make up the activity). (Note: The latter helped us establish the $5^{\text {th }}$ functional requirement.)

- The audio and images for the prompts and reinforcers were set to load before the activity begins in order to minimize the lagging that tends to occur while it is in progress (e.g., user's responses were either not detected or processed so slowly that they would not receive feedback regarding the correctness of their selections in a timely manner) if the strength and speed of the internet connection is weak or unstable. (Although we thought the issue had been resolved, we learned during user evaluations that the issue persisted.)

Next, in collaboration with Imagine!, we ran preliminary user evaluations of the prototype at their care providing facilities, including their smart group homes, with some of their clients with DD (primary users) and their caregivers or guardians (secondary users). Primary users were asked to try each activity (once) and to answer a set of follow-up questions after trying each one. Secondary users were asked to help researchers: 1) administer the activities to users, 2) make observations about users' interaction with the activities, and 3) answer questions about users' interaction with the activities. In order to avoid making the preliminary user evaluations too strenuous for primary users, the activities were divided into three groups and each group was evaluated on a different day: First, the Numbers, Colors, and Money activities were tested by seven individuals with DD aged 28-53 years old. Next, the Shapes and MA activities were tested by five individuals with DD aged 30- 58. Finally, the LL and UL activities were tested by three individuals with DD aged 39-45. Overall, the feedback sought and the follow-up questions was qualitative in nature and it consisted of how users interacted with and reacted to the activities and, from there, the improvements that needed to be made to the activities with regards to their UI, usability, and functionality in order for them to be more effective and provide a better user experience. The main findings were:

- The activities and the positive and learning reinforcers were well-received by participants.

- All the participants were able to complete the activities with little to no assistance.

- Participants found the touch-screen interface easier to use than paper when doing similar activities because of how easy it is to respond to prompts with the app (e.g., with paper they would have to write their answers).

- Improvements that needed to be made to the system:

- Make the detection of user input (via touch or stylus) faster and/or more reliable.

- Speed up loading times and response-time (i.e., make activities respond to users' input more quickly).

- Add audio signals for when the activities start and end.

- For the MA, make the audio of the target total more accessible (i.e., make button easier to see and press) and make the hints about which currency cannot be used to make up the total more obvious (i.e. grey it out).

- Ensure content displays properly in Portrait mode.

On another note, after stage 1, we found that to get a better idea of participants' interactions with the activities, we needed to ask caregivers more direct questions and some of the questions for the participants needed to be rephrased or omitted. So, we applied this to stages 2 and 3 of the preliminary user evaluations.

\subsection{User Study and Results}

Once the system was improved based on the results from the preliminary user evaluations, we ran a more comprehensive user study of the system, in collaboration with Hope Services, with ten of their clients with DD at one of their care providing facilities which offers day-programs. Participants, four males and six females, were between the ages of 23 and 36 (mean = 28.4). A summary of participants' demographics is shown in Table 1.

Table 1. Summary of participants' demographics

\begin{tabular}{cccl}
\hline ID & Age & Gender & Disability \\
\hline P1 & 23 & M & Cerebral Palsy \\
P2 & 31 & M & Autism \\
P3 & 25 & F & Inverted X Syndrome \\
P4 & 36 & F & Down Syndrome \\
P5 & 27 & F & Cognitive/Intellectual Disability \\
P6 & 35 & F & Mild Intellectual Disability \\
P7 & 27 & F & Mild Intellectual Disability \\
P8 & 24 & M & Autism \\
P9 & 26 & M & Mild Intellectual Disability \\
P10 & 30 & F & Down Syndrome \\
\hline
\end{tabular}

Participants were asked to try each activity on three different sessions over the course of a week and, after each session, to answer a set of follow-up questions that aimed to assess their experience with the activity and their feelings towards it. In order to avoid making the evaluations too strenuous, participants were asked to try three activities (picked and ordered randomly) one week and the remaining four activities the next week (ordered randomly again). Nine participants tried all the activities this way, except for P9 who completed the last session of the first three activities he/she was asked to try on the first week during the second week. The remaining participant (P10) only tried three activities: LL, Money, and Numbers.

Noting that our target users tend to be less critical and more positive when giving feedback, we also gathered user feedback through observations of the sessions. With consent from the participants and Hope Services, we recorded videos of all the session and audio of participants' responses to the follow-up questions. In addition, the activities recorded participants' (incorrect or correct) responses to prompts. Hence, the data gathered through the user study is both quantitative and qualitative in nature. Consequently, data analysis involved a mixed method approach. As a first step, in order to analyze the videos of the sessions through content analysis, four researchers went through the videos, noted the following themes, and defined a coding scheme based on them: 
- Positive reaction: Physical (e.g., smile, laugh) or verbal indications that participant is enjoying the activity.

- Negative reaction: Physical or verbal indications that participant is not enjoying the activity (e.g., facial or physical signs of frustration or disinterest).

- Assistance: Participant needed assistance from researchers to perform specific actions without which they would not have been able to continue/complete the activity.

- $\quad$ Multiple presses to make a selection (MP): Participant had to press an option more than once to make a selection.

Three researchers then independently coded the same 8 videos (i.e., quantified the occurrences of the aforementioned themes) and obtained an acceptable interobserver agreement (90.5\%). All the videos (198 in total) were then divided among the three researchers and coded independently. Video coding was done using the Behavioral Observation Research Interactive Software (BORIS $^{6}$ ), an easy-to-use event logging software for video/audio coding of living observation. Data from the audio recordings was quantified when possible. Finally, all the quantitative data was analyzed using descriptive statistics, mainly summary statistics.

\subsubsection{Results}

Here we present our results from the analysis of the quantitative and qualitative data concerning participants' interactions with the activities. Due to space limitations, results will be presented in the following groups and order that follow: 1) Colors, Money, Numbers, and Shapes since they behave similarly. 2) LL and UL since they reinforce the general skill of recognizing letters and behave similarly. 3) MA will be discussed independently because it aims to reinforce a more advanced skill, plus it behaves a bit different from the other activities.

\subsubsection{Colors, Money, Numbers, and Shapes}

The Colors and Shapes activities were tested by nine participants, while the Money and Numbers activities were tested by all ten participants. Here we present all the data gathered while participants tried these activities.

1. Positive and negative reactions: A summary of the reactions displayed by participants (who displayed any reaction) while trying these activities is shown in Figure 5. Their reactions are further summarized below. On average:

- Colors: $83.9 \%(\mathrm{SD}=0.34)$ of their reactions were positive and $16.1 \%(\mathrm{SD}=0.34)$ were negative.

- Money: 95.8\% (SD = 0.12) of their reactions were positive and $4.2 \%(\mathrm{SD}=0.12)$ were negative.

- $\quad$ Numbers: $95.8 \%(\mathrm{SD}=0.06)$ of their reactions were positive and $4.2 \%(\mathrm{SD}=0.06)$ were negative.

- Shapes: 97.1\% (SD $=0.05)$ of their reactions were positive and $2.9 \%(\mathrm{SD}=0.05)$ were negative.

While trying all four activities, P7 did not display any reactions. Likewise, P10 did not display any reactions while trying the Money and Numbers activities.

2. Assistance needed from researchers: P2 was the only participant who displayed this theme while trying all four activities: six times with Colors (during the third session) and Money (during S1), four times with Numbers (during S2), and one time with Shapes (during S1).

3. Incorrect responses: The total number of times each participant responded incorrectly to a prompt while trying

\footnotetext{
${ }^{6} \mathrm{http}: / /$ penelope.unito.it/boris/
}

these activities is shown in Table 2 (Rows $\mathrm{C}, \mathrm{M}, \mathrm{N}$, and $\mathrm{S}$ ). P7 did not make incorrect responses during these activities.

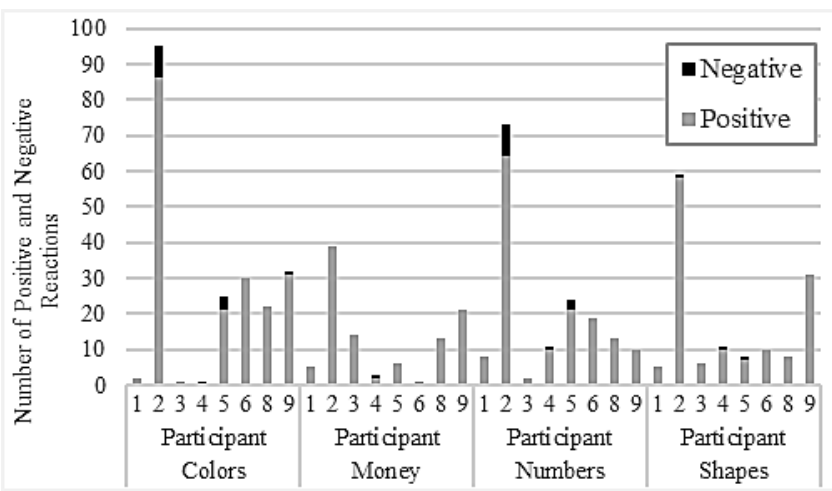

Figure 5. Positive and Negative Reactions with the Colors, Money, Numbers, and Shapes activities.

Table 2. Total number of incorrect responses

\begin{tabular}{|c|c|c|c|c|c|c|c|c|c|}
\hline & P1 & P2 & P3 & P4 & P5 & P6 & P8 & P9 & P10 \\
\hline $\mathrm{C}$ & 1 & 0 & 0 & 0 & 0 & 0 & 0 & 1 & \\
\hline $\mathrm{M}$ & 3 & 3 & 2 & 3 & 1 & 0 & 1 & 3 & 3 \\
\hline $\mathrm{N}$ & 0 & 0 & 0 & 0 & 1 & 0 & 0 & 0 & 0 \\
\hline $\mathrm{S}$ & 7 & 1 & 0 & 8 & 0 & 0 & 0 & 7 & \\
\hline LL & 4 & 0 & 2 & 2 & 0 & 1 & 0 & 5 & 2 \\
\hline UL & 0 & 2 & 0 & 1 & 0 & 1 & 0 & 4 & \\
\hline
\end{tabular}

Colors (C), Money (M), Numbers (N), and Shapes (S).

1. MP: The total and average number of instances of MP experienced by participants while trying these activities is shown in Table 3 (Rows $\mathrm{C}, \mathrm{M}, \mathrm{N}$, and $\mathrm{S}$ ). When researchers noted that many instances of MP occurred, they would ask participants if they were frustrated by that. Their responses are summarized in Table 4.

Table 3. Total number of instances of MP

\begin{tabular}{|c|c|c|c|c|c|c|c|c|c|c|c|}
\hline & P1 & P2 & P3 & P4 & P5 & P6 & P7 & P8 & P9 & P10 & Avg \\
\hline $\mathrm{C}$ & 25 & 18 & 1 & 20 & 5 & 2 & 10 & 14 & 11 & & 11.78 \\
\hline M & 7 & 11 & 2 & 10 & 3 & 1 & 3 & 10 & 10 & 3 & 6.00 \\
\hline $\mathrm{N}$ & 14 & 16 & 0 & 6 & 10 & 3 & 10 & 4 & 13 & 1 & 7.70 \\
\hline $\mathrm{S}$ & 20 & 8 & 2 & 7 & 3 & 2 & 12 & 9 & 10 & & 8.11 \\
\hline LL & 9 & 15 & 3 & 10 & 1 & 12 & 7 & 12 & 14 & 6 & 8.90 \\
\hline UL & 6 & 18 & 0 & 21 & 3 & 6 & 6 & 16 & 8 & & 9.33 \\
\hline
\end{tabular}

Table 4. Participants' responses to the question regarding MP being frustrating

\begin{tabular}{c|ccccccccc}
\hline Response & $\mathbf{C}$ & $\mathbf{M}$ & $\mathbf{N}$ & $\mathbf{S}$ & $\mathbf{L L}$ & $\mathbf{U L}$ & $\mathbf{M A}$ & $\mathbf{T}$ & $\%$ \\
\hline Yes & 1 & 1 & 1 & 1 & 0 & 0 & 3 & 7 & $35 \%$ \\
No & 1 & 1 & 4 & 1 & 2 & 0 & 4 & 13 & $65 \%$ \\
\hline
\end{tabular}

Colors (C), Shapes (S), Money (M), Numbers (N), and Total (T).

2. Responses to follow-up questions: The main follow-up questions asked along with participants' responses regarding these activities are summarized in Table 5 (Columns C, M, $\mathrm{N}$, and $\mathrm{S}$, next page). The participant for whom the Money activity was not easy (P2) said it was hard because the iPad was not detecting his/her selections (input) properly. 
Table 5. Summary of questions and participants' responses

\begin{tabular}{ccccccccc}
\hline Question & Response & $\mathbf{C}$ & $\mathbf{M}$ & $\mathbf{N}$ & $\mathbf{S}$ & $\mathbf{L L}$ & $\mathbf{U L}$ & MA \\
\hline \multirow{2}{*}{ Easy? } & $Y$ & 9 & 9.7 & 10 & 9 & 9 & 9 & 8.7 \\
\cline { 2 - 8 } & $N$ & 0 & 0.3 & 0 & 0 & 1 & 0 & 0.3 \\
\hline \multirow{2}{*}{ Fun? } & $Y$ & 9 & 10 & 10 & 9 & 10 & 9 & 8.7 \\
\cline { 2 - 8 } & $N$ & 0 & 0 & 0 & 0 & 0 & 0 & 0 \\
\hline \multirow{2}{*}{ Helpful? } & $Y$ & 9 & 10 & 10 & 9 & 10 & 9 & 9 \\
\cline { 2 - 8 } Liked it? & $N$ & 0 & 0 & 0 & 0 & 0 & 0 & 0 \\
\cline { 2 - 8 } & $Y$ & 9 & 10 & 10 & 9 & 10 & 9 & 9 \\
\hline \multirow{2}{*}{ Use again? } & $Y$ & 0 & 0 & 0 & 0 & 0 & 0 & 0 \\
\cline { 2 - 8 } & $N$ & 0 & 0 & 0 & 0 & 0 & 0 & 0 \\
\hline Liked & $Y$ & 8 & 9 & 10 & 8 & 10 & 9 & $8.5^{*}$ \\
\cline { 2 - 8 } reinforcers? & $N$ & 0 & 0 & 0 & 0 & 0 & 0 & $0.5^{*}$
\end{tabular}

* P3's answer was coded as "Y and N" since P3 expressed that she liked the positive reinforcers but not the learning reinforcers while trying the MA activity. Thus, 0.5 was assigned to both ' $\mathrm{Y}$ ' and ' $\mathrm{N}$ '.

Note: Not all participants were asked or responded to these questions. If a question was asked during each session and participants' responses differed across sessions, the average was computed.

\subsubsection{Lowercase Letters and Uppercase Letters}

The LL activity was tested by all ten participants, while the UL activity was tested by nine participants. Here we present all the data gathered while participants tried these activities.

1. Positive and negative reactions: A summary of participants' reactions (if they displayed any reaction) while trying these activities is shown in Figure 6. Their reactions are further summarized below. On average:

- LL: $89.5 \%(\mathrm{SD}=0.31)$ of their reactions were positive and $10.5 \%(\mathrm{SD}=0.31)$ were negative.

- $\quad \mathrm{UL}: 97.9 \%(\mathrm{SD}=0.04)$ of their reactions were positive and $2.1 \%(\mathrm{SD}=0.04)$ were negative.

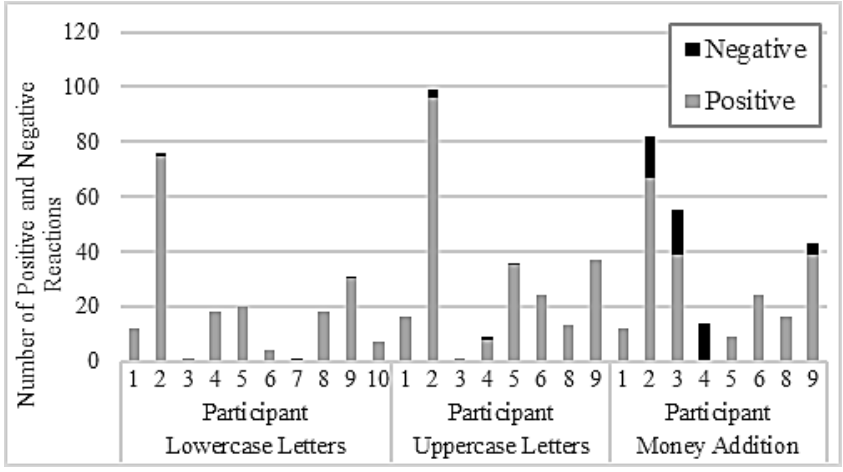

Figure 6. Positive and Negative Reactions with the LL, UL and MA activities.

2. Assistance needed from researchers: P1 was the only participant who displayed this theme while trying the LL activity: once during S1 and another time during S2.

3. Incorrect responses: The total number of times each participant responded incorrectly to a prompt while trying these activities is shown in Table 2 (Rows LL and UL). P7 did not make incorrect responses during both activities.

4. MP: The total and average number of instances of MP experienced by participants while trying these activities is shown in Table 3 (Rows LL and UL). When researchers noted that many instances of MP occurred, they would ask participants if they were frustrated by that. Their responses are summarized in Table 4.

5. Responses to follow-up questions: The main follow-up questions asked along with participants' responses regarding these activities are summarized in Table 5 (Columns LL and UL). The participant who expressed that the LL activity was not easy (P1) explained that finding letters was hard because there were too many letters and they were unordered.

\subsubsection{Money Addition}

The MA activity was tested by nine participants. Here we present all the data gathered while participants tried these activities.

1. Positive and negative reactions: A summary of the reactions displayed by participants (who displayed any type of reaction) while trying this activity is shown in Figure 6 (right). Their reactions can be further summarized as follows. On average, $80.4 \%(\mathrm{SD}=0.34)$ of their reactions were positive and $19.6 \%(\mathrm{SD}=0.34)$ were negative.

2. Assistance needed from researchers: Four participants displayed this theme while trying the MA activity: P1 four times (two times during both S1 and S2), P2 twenty-three times (twelve times during S1, five times during S2, and six times during S3), and P4 and P6 one time (both during S1).

3. MA responses: Participants responses (i.e., the total number of bills and coins they pressed to get to the target total) were compared to the optimal responses (i.e., the minimum number of bills and coins needed to get to the target total) by dividing their responses by the optimal ones. The average of these comparisons are shown in Table 6. So, for example, on average, P1 would use 1.4 times the minimum number of bills and coins needed to get to the target total.

Table 6. Participants' responses versus the optimal responses

\begin{tabular}{c|ccccccccc}
\hline \multicolumn{1}{c}{} & P1 & P2 & P3 & P4 & P5 & P6 & P7 & P8 & P9 \\
\hline Average & 1.4 & $\mathbf{2 . 2}$ & $\mathbf{2 . 4}$ & $\mathbf{2 . 4}$ & $\mathbf{1 . 9}$ & $\mathbf{2 . 2}$ & 1.1 & 1 & 1.1 \\
SD & 0.6 & 0.7 & 0.8 & 1 & 0.4 & 0.7 & 0.2 & 0 & 0.1 \\
\hline \multicolumn{8}{c}{ Standard deviation (SD). }
\end{tabular}

6. Strategies: Based on the videos, we observed that four participants (P1-P4) seemed to normally choose bills and coins at random, three participants $(\mathrm{P} 7, \mathrm{P} 8, \mathrm{P} 9)$ seemed to closely follow the optimal strategy, and the remaining two (P5, P6) seemed to normally touch bills and coins in order.

7. Responses to follow-up questions: The main follow-up questions asked along with participants' responses regarding the MA activity are summarized in Table 5 (Column MA). The participant who said that the MA activity was not easy during S1 (P1), expressed in later sessions that it got easier.

\section{Discussion}

From the user studies we gathered that all the activities were wellreceived since all the participants made positive remarks about each of the activities (e.g. they were fun, easy, helpful, and they liked them) and, on average, $80.4 \%$ of their reactions (or greater) were positive. This is in spite of the negative reactions displayed by participants while trying the activities, which researchers noted occurred generally when participants experienced instances of MP. On that note, all participants experienced instances of MP while trying almost all of the activities. When asked if those instances (of MP) were frustrating, $65 \%$ of the time participants expressed that those instances were not frustrating. Hence, despite 
its flaws (i.e., lagging and slow input detection that seems to occur when the internet connection is not $\operatorname{good}^{7}$ ) due to it being a web-app, our system has the potential to be well-received by target users and to help make the experience of reviewing basic skills easy and enjoyable for people with DD.

We also gathered that most participants were able to complete the activities on their own (with little to no assistance from researchers). Still, one participant in particular (P2) needed assistance from researchers while trying most of the activities (with five out of the seven activities). In addition, while trying the MA activity, many participants needed assistance from researchers (four out nine participants). Thus, the MA activity needs to be improved (by providing further guidance) so that users need little to no assistance from caregivers while doing this activity. With all the other activities, at most one participant needed assistance from researchers. Still, results suggest that the proposed system of activities has the potential to increase users' independence when doing these type of activities (while also decreasing the workload of their caregivers). That is, results suggest that our system has the potential to minimize how much help users need from their caregivers while completing these types of activities (i.e., caregivers will not need to be there all the time to say all the prompts that make up the activities and to tell them if their answers are correct or not).

Furthermore, we gathered from the user study that:

- The reinforcers seemed to serve their purpose since most participants expressed that they liked the reinforcers and that the learning reinforcers were helpful.

- Using pictures of things that are generally well-liked (e.g. sports, animals) will likely serve as good positive reinforcers.

- The following improvements can also be made:

- Adding new activities: Activities that do not prompt users and allow them to review numbers, colors, letters, shapes, and money more freely. Also, activities that help users review more than one skill at a time (e.g. an activity that prompts the user to "Press the red three.") or more advanced skills like budgeting money.

- Making MA activities more "real" by providing varying amounts of bills and coins and by allowing users to subtract from the gathered amount. It can also be made to allow users to turn on/off the learning reinforcers (i.e., allow users to decide whether they will hear audiofeedback regarding their selections within this activity).

- For both activities related to money, make the pictures of the coins more truthful to their true size, especially in terms of the relative size between coins (e.g., the dime should be smaller than the nickel).

- Increase difficulty as users progress through the activities; e.g., randomize the order of the letters and/or increase the number of letters, review bigger numbers, more shapes and colors, etc.

- Add pictures of other things that are generally wellliked by most people (e.g., other sports, animals, race cars, superheroes, etc.).

\section{Conclusions and Future Work}

We present the development and evaluation of an online learning system for people with developmental disabilities (DD) of all

\footnotetext{
7 Note: The wireless internet connection at the testing site was visibly slower than in our lab when loading some websites, so our best guess is that this caused the lagging.
}

ages. Overall, through the user study we gathered that, despite the improvements that need to be made and the system's inherent flaw as a web-app (i.e., its dependence on the strength and speed of the internet connection) and the issues that may arise because of that, our system has the potential to be a helpful learning tool for people with DD reviewing basic skills and for caregivers helping people with DD review basic skills.

From our preliminary (user) evaluations we learned how the system could be improved, but we were not able to implement some of the improvements since the system is a web-app that uses many media files (which means its use is limited to places with good internet connections). Then, through a comprehensive user study with individuals with DD we gathered that, despite the system's inherent flaws and issues due to it being a web-app and the improvements that need to be made, results suggest that the proposed system will likely be well-received by target users and that it has the potential to help: 1) make the experience of reviewing basic skills easy and enjoyable for people with DD, and 2) increase users' independence while doing the activities to review basic skills and, as a result, lessen their caregivers workload when they are trying to help them review basic skills. In summary, after making the necessary (and possible) improvements, we are confident that the proposed system will have the potential to be a practical and helpful learning tool for people with DD who need to reinforce basic skills.

One of the main contributions of our work lies in our methods for gathering feedback from people with DD. By combining debriefing interviews and an analysis of participants' behaviors during evaluations, we were able to gather a better assessment of participants' experiences and reactions to our system despite target users' tendency towards providing positive feedback.

The next steps for this project include: 1) improving the system based on the feedback we got from the user study (e.g., we will include a collection of stock photos and videos in various categories that will serve as positive reinforcers and we will allow users to choose the category of the positive reinforcers they see), 2) adding more activities that cover things like budgeting money, telling time, and recognizing street signs, common objects, and concepts with regards to object-relations (e.g., shorter, longer), and 3) evaluating the effectiveness of the activities by running a long-term user study ( 2 weeks) where participants will be asked to use the activities while we track their progress. In relationship to the latter, we conducted a preliminary assessment of the long-term effect of our system on the motivation of people with DD to reinforce basic skills by contacting our collaborating organizations (at least 4 weeks after the user study ended) and asking them whether users/caregivers were still using our system. The one collaborating organization who replied informed us that some of their clients were still using the system.

\section{Acknowledgments.}

This material is based upon work supported by the National Science Foundation through the Graduate Research Fellowship (grant No. DGE-1339067) and the UC MEXUS-CICESE scholarship. We would like to thank Imagine! Colorado and their clients as well as Hope Services and their clients for their help with the user evaluations. We would also like to thank Luke Buschmann for his crucial help with the development and evaluation of the Money, Colors, and Numbers activities; Mike Tan for his crucial help with the development of the Money Addition and Shapes activities, and Sonya Pita for her help with the preliminary evaluations, the analysis of the preliminary user evaluation results, and the development of the letters activities. 


\section{References}

[1] Alberto, P. A., Cihak, D. F., \& Gama, R. I. (2005). Use of static picture prompts versus video modeling during simulation instruction. Research in Developmental Disabilities, 26, 327-339.

[2] Canella-Malone, H., Sigafoos, J., O'Reilly, M., de la Cruz, B., Edrisinha, C., and Lancioni, G. E. (2006). Comparing video prompting to video modeling for teaching daily living skills to six adults with developmental disabilities (Doctoral dissertation).

[3] Furniss, F., Lancioni, G., Rocha, N., Cunha, B., Seedhouse, P., Morato, P., \& O'Reilly, M. F. (2001). VICAID:

Development and evaluation of a palmtop-based job aid for workers with severe developmental disabilities. British Journal of Educational Technology, 32(3), 277-287.

[4] Goodson, J., Sigafoos, J., O’Reilly, M., Cannella, H., and Lancioni, G. E. (2007). Evaluation of a video-based error correction procedure for teaching a domestic skill to individuals with developmental disabilities. Research in Developmental Disabilities, 28(5), 458-467.

[5] Kagohara, D. M., van der Meer, L., Ramdoss, S., O’Reilly, M. F., Lancioni, G. E., Davis, T. N., Rispolie, M., Langb, R., Marschikg, P. B., Sutherlandh, D., Green, V. A., and Sigafoos, J. (2013). Using iPods and iPads in teaching programs for individuals with developmental disabilities: A systematic review. Research in developmental disabilities, 34(1), 147-156.
[6] Lancioni, G. E., O’Reilly, M. F., Seedhouse, P., Furniss, F., and Cunha, B. (2000). Promoting independent task performance by persons with severe developmental disabilities through a new computer-aided system. Behavior Modification, 24(5), 700-718.

[7] Morales-Villaverde, L. M., Smith, S. R., and Kurniawan, S. (2013, October). Brain-training software for stroke survivors. In Proceedings of the 15th International ACM SIGACCESS Conference on Computers and Accessibility (p. 40). ACM.

[8] Sigafoos, J., O’Reilly, M., Cannella, H., Upadhyaya, M., Edrisinha, C., Lancioni, G. E., Hundley, A., Andrews, A., Garver, C. and Young, D. (2005). Computer-presented video prompting for teaching microwave oven use to three adults with developmental disabilities. Journal of Behavioral Education, 14, 189-201.

[9] Sturmey, P. (2003). Video technology and persons with autism and other developmental disabilities: An emerging technology for PBS. Journal of Positive Behavior Interventions, 5, 3-4.

[10] Wehmeyer, M. L., Palmer, S. B., Smith, S. J., Parent, W., Davies, D. K., and Stock, S. (2006). Technology use by people with intellectual and developmental disabilities to support employment activities: A single-subject design meta analysis. Journal of Vocational Rehabilitation, 24(2), 81-86.

[11] Wiemeyer, J. and Kliem, A. (2012). Serious games in prevention and rehabilitation - a new panacea for elderly people?. European Review of Aging and Physical Activity, 9(1), 41-5. 